\title{
ROLE OF HEAVY METAL SALTS IN PROTECTION OF VIGNA MUNGO (L.) HEPPER SEEDLINGS AGAINST ALTERNARIA LEAF SPOT INFECTION BY
}

\section{SEED TREATMENT}

\author{
M. N. Bhajbhuje \\ Jawaharlal Nehru Mahavidyalaya, Wadi, Nagpur-440 023 (M.S.) \\ Corresponding author Email : dr_mnbhajbhuje@rediffmail.com
}

\begin{abstract}
The phytoalexins involvement in disease resistance against virulent pathogens is being widely realizing in different plant system. The salts of heavy metals, well-known phytoalexin inducers, were used at dilute concentrations in different treatments to control Alternaria leaf spot infection in Vigna mungo (L.) Hepper seedlings caused by necrotrophic fungal pathogen Alternaria alternata Keissler. Seed soaking for $24 \mathrm{~h}$ induced significant to moderate protection to seedlings, at different stages of growth after treatment, against artificial inoculation of spore suspension of Alternaria alternata Keissler an isolate of virulent pathogen and mostly induced significant reduction in symptoms. Seed soaking in aqueous dilute solutions of test chemicals comparatively seemed to provide the most effective and durable protection. Wet seed treatment with barium, mercury and ferric chloride were proved most effective in reducing the infection and lesion expression significantly. The fungitoxicity in leaf diffusate was recorded decline with age in treated artificially inoculated seedlings. Little fungitoxicity appeared in leaf diffusates from 4-wk old seedlings that had been treated uninoculated and moderate toxicity in that from untreated, inoculated seedlings. However, treated seedlings inoculated at 3-and 4-wk stage produced leaf diffusates with significantly greater toxicities which were in proportion to their abilities to resist attack.
\end{abstract}

\section{Keywords}

Vigna mungo (L.) Hepper heavy metal salts, leaf diffusates, phytoalexin, resistance,

\section{Introduction}

Vigna mungo (L.) Hepper, a fast growing herbaceous legume pulse of Central Asia and Indian origin, belongs to family Fabaceae in recorded history, is extensively cultivated as staple food crop in the southern parts of Asia, Africa and Madagascar for its energizer nutritious beans as the seeds are excellent source of easily digestible plant protein (26\%); carbohydrate; minerals; vitamin- 
A, B1, B2, Niacin; lesser content of fat; zero cholesterol \& glycosides. It is a store house of dietary fibers, calcium, potassium, iron, magnesium and copper (Savithsuri, 2013). Globally black gram accounts for over $40 \%$ of total legume seed traded. India is leading producer and consumer of black gram seeds on the globe, contributing about 1.5 million tons of global annual output whereas Myanmar and Thailand are major exporters (Sharma et al, 2011). Lion"s share of India"s production, accounting for over $60 \%$ of the nation"s total output is contributed by South India (Wikipedia, 2014).

\section{Material and Method}

Vigna mungo (L) Keissler is used as experimental material. The fungitoxicity of an aqueous solution in dilute concentration (10-2 to $10-5 \mathrm{M})$ of six salts of heavy metal of diverse nature was assayed for their toxic response on spore germination of Alternaria alternata Keissler employing spore germination technique (CIM, 2010). The concentration (10-4 M) was seemed to be most effective in inhibition of spore germination and germ tube growth, was selected for further treatment. Seeds were soaked for 24 hour in test solution (10-4 M) and being sown in sterile soil supplemented with FYM in $16 \mathrm{~cm}$ earthen pots. The pots were maintained in laboratory at $16-280 \mathrm{C}$, where sufficient light is available and watered daily.

\section{Result and Discussion}

Six heavy metal salts were screened to study the parameters as under: Initially, in vitro fungitoxicity of test chemicals was assayed and results are presented in table 1 In-vitro fungitoxicity of test chemicalsTable 1 : Effects of heavy metal salts on spore germination \& germ tube growth of Alternaria alternata KeisslerChemical Conc. : Direct toxicity of heavy metal salts of varying origin to the fungal pathogen does not seem to explain the reduction of symptoms. Chlorides of ferrous and barium are non-toxic, provided stronger protection than mercuric, cadmium and cupric chloride, a highly toxic one. These test 
chemicals may exert inhibitory influence upon fungal spores germination and impose upon them exogenous dormancy. This is clearly shown by sensitivity of fungal spores to chemicals by several researchers. The inhibition of spore germination may be attributed to variable toxic effect of test chemicals. Similar findings were reported with conidia of Alternaria tenuis (Bhajbhuje, 1989), A. alternata (Ashraf and Ali, 2007), A. porae (Feofilova et al., 2012), A. solani (Bhajbhuje, 2013). The hydrolytic products of the chemicals possibly at low conc. induced dormancy or may cause injuries to fungal spores by dissolving the protective thick wall layers and plasma membrane or ruptured them making porous.Lesion number was reduced significantly in young seedlings in most of the treatments, the greatest effects being recorded with chlorides of copper, mercury and cadmium, reducing the lesion number at 2-wk stage to the extent of 71 to $84 \%$. Such induced inhibitory effects gradually declined to 49 to $63 \%$ in 4 -wk stage except chlorides of mercury and barium, while lesion expansion was inhibited in all cases, there were proportionately more small lesions and fewer large lesions. The stronger inhibition was recorded with chlorides of mercury, copper and barium (Table 2).The seed soaking in aqueous solution of test chemicals seemed to be the most effective for most of the treatment, induced $61-86 \%$ protective effect at 3 -wk stage. The inhibitory effect gradually declines in most of the treatment with age of plant except barium chloride. A greatest inhibition was recorded at 2-wk growth stage to the extent of 60 to $82 \%$ in the seedling receiving seed treatment. The seed treatment provide substantial protection and long persistent of such effect at significant level. These results are confirmed with earlier finding in Solanum melongena L (Bhajbhuje, 2013). The accumulation of phytoalexins in response to chemical seed treatment was confined in peanuts (Sobolev et al., (2007).Persistence of protective effectMoreover, substantial reduction in lesion number was recorded for all the treatments at 5-wk stage, the most pronounced effects being recorded with barium chloride reducing lesion number by $76 \%$ followed by chloride of mercury and copper while, other 
treatment had moderate protective effect, reducing the lesion number to the extent of $40 \%$ to $57 \%$, while lesion expansion was inhibited in all cases, stronger inhibition was recorded with chlorides of barium, mercuric and cadmium. Substantial protective effect was also recorded for 6-wk-old inoculated plants with most of the treatments, causing $29-57 \%$ inhibition in this respect. Greatest effect was particularly significant with chlorides of barium and mercury followed by cadmium and cupric chloride. The lesion expansion was significantly inhibited and lesion number was appreciably reduced in treated inoculated seedlings. In such treatments, there were proportionately more small lesions and fewer large lesions compared with those in control. The reduction in number of infections in most of the treatments and the inhibition of lesion expansion in some, suggesting that the induced resistance may have operated at two stages i.e., initially by limiting the number of successful infection and subsequently by restricting lesion size. This may imply some post inflectional changes in the leaf tissue in treated plants that may limit the in vitro activity of the pathogenFungitoxicity of leaf diffusate:In the final experiment, the seedlings were raised in pots from soaked seeds and inoculated at the age of 3-wk. Leaf diffusates were obtained initially from 2-wk old uninoculated plants and later from both uninoculated and inoculated plants in different treatments 3 days later each inoculation and bio-assayed for fungitoxicity (Table 3). Leaf diffusates collected from uninoculated plants in different treatments elicited supportive response to spore germination with very mild toxic effect. However, diffusates from comparable inoculated plants in all treatment induced significant levels of fungitoxicity, causing 61 to $83 \%$ spore inhibition compared to only $12 \%$ in control at 3 -wk stage. Some relation was evident between each level of toxicity $\&$ degree of resistance. Most pronounced toxicity was recorded with chlorides of mercury, copper $\&$ barium while a moderate toxicity was confined with lithium sulphate over control. The leaf diffusates from 4-wk-old inoculated plants was seemed to be comparatively less fungitoxic, causing $37-75 \%$ reduction in spore germination compared to only 
$7 \%$ reduction in control indicated fungitoxicity of leaf diffusates gradually declines with age of the plant (Table 3). Leaf diffusates from uninoculated plants in different treatments supported germ tube growth almost to the same extent inducing very little toxic effect. However, diffusates from inoculated plants induced significant levels of fungitoxicity, reducing germ tube growth by 64 to $84 \%$, compared to only $14 \%$ reduction in control at 3-wk stage. A greatest toxicity was reported at this stage with chlorides of mercury and barium, considerable with cadmium \& ferric chloride while others had moderate toxic effect on germ tube growth. A little toxicity (2-5\%) was noted with leaf diffusates from 4-wk-old uninoculated plants in treatments with chlorides of cupric, ferric and cadmium while mild toxicity, 1-2\% was recorded for others compared to $46-74 \%$ reduction in germ tube growth in inoculated treated plants (Table 3)Inoculation with compatible race of pathogen itself led to the development of considerable fungitoxicity in plants grown from untreated seeds. This seems to have resulted from production of antifungal compounds in the leaves themselves by plant during successive growth period. Battu et al., (2011) reported some antifungals, glycolalkaloid, phenol, ononitol, omethylscyllo-inositol from leaves of Vigna mungo L. Pathogenesis in leaves exhibited antimicrobial activity (Karthikeyan et al., 2009). The volatile extract of blackgram leaves reported antifungal, induced total inhibition of mycelial growth \& sporulation of indicating a defense response against these pathogenic fungi (Battu et al., 2011). Reduced fungitoxic effect of leaf diffusates on both spore germination and germ tube growth was recorded with all plants receiving treatment, when inoculated 3 days after 3 -wk growth stage with spore suspension of Alternaria alternata. The leaf diffusates from 3-wk old inoculated plants receiving treatment with chlorides of mercury and barium were reported comparatively effective, causing highest reduction in spore germination followed by cupric chloride while others had moderate inhibitory effect. The leaf diffusates from plants receiving these treatments were highly toxic to pathogen, reducing $63-82 \%$ germ tube growth. A fungitoxicity was gradually decline 
with age of plants. A leaf diffusates of 4-wk-old inoculated plants receiving seed treatment with all chlorides except cadmium chloride and lithium sulphate was reported considerably toxic to pathogen, inhibiting $68-75 \%$ spore germination as well as $69-74 \%$ germ tube growth while other treatments induced moderate toxicity for these parameters (Table 3)Significant to moderate levels of fungitoxicity was recorded in the leaf diffusates from 3-wk-old uninoculated seedlings in different treatment while considerable to mild toxicity wasobserved from 4-wk-old seedlings receiving treatment with chlorides of mercury, barium and copper while mild toxicity was recorded with others treatments. The fungitoxicity rapidly declined with plants age and disappear between 5-6-wk in most of the treatments. It is possible that the substance was metabolized and also diluted by plant growth so as to leave moderate toxic effect at the 4-wk stage. The untreated (control) plants themselves developed considerable, 7-13\% fungitoxicity when inoculated after 3- or 4-wk. The greater post-infection development of fungitoxicity in the seed treated plants, even 4-wk, after seed treatment, appears to be more significant than the initial development of toxicity in them. This seems to have resulted from the interaction between seed treatment and infection, mediated possibly through some alteration in host metabolism. These results are confirmed with earlier reports as in Peanut (Sobolev et al., 2007); Cicer arietinum L (Raju et al, 2008); Fragaria vesca (Amil-Ruiz, et al., 2011). Bhajbhuje (2013) reported similar finding while studying the role of phytoalexin inducer chemical in Solanum melongena $\mathrm{L}$. The results obtained in the present investigation reveals that in vitro, chlorides of mercury, copper and cadmium inhibited total spore germination and germ tube growth but allow mild lesion formation in 5-6-wk old plants. At this stage of growth, moderate toxicity was evident in leaf diffusate of plants from any treatment. This may suggests that some quantity fungitoxic substance was produced in Vigna mungo in response to seed treatment. Little symptoms were produced when the production of fungitoxic substances soon attains a level inhibitory to the invading organism. Pronounced inhibition of germ tube growth 
in some treatments in relation to spore germination seemed to be of particular significance when such toxicity is confined relating to disease resistance as a post-interaction phenomenon. Seed treatment interfered with infection process itself, since barium and mercuric chloride induced significant reductions in the number of lesions, and as well as mean lesion size implying appreciable inhibition of lesion number was less pronounced but that on lesion size appeared to be greater for most of the treatments where plants exhibited proportionately more small lesions and fewer large lesions as compared with untreated plants. These results coincided with earlier findings with Solanum melongena L. (Bhajbhuje, 2013).The symptom expression was significantly inhibited at $2-3 w k$ and moderately at $4-5 w k$ stages in most of the seed treatments. The protective effect of seed treatment was persisted in 6-wk-old plants but it was declined by $5-8 \%$ over 5 -wk-old plants with all the treatment. This may be quite possible that production of phytoalexin-like substances was initially stimulated and detected in young seedlings but at later stages, there may be insufficient quantity of accumulation of phytoalexins-like substances by little aged seedlings (Eckadt, 2011) or applied chemicals may have been metabolized in host tissues and their concentrate probably were diluted by seedling growth so as to leave little toxic effect at later stages (Bhajbhuje, 2013). It seems unlikely that as the chlorides of mercury and barium had their direct toxic effect on the pathogen but these test chemicals have provided substantial protection to Vigna mungo seedlings; it was in all probability an induced effect. Pronounced production of fungitoxic substance in the treated plants in different treatments when inoculated may induced moderate to high level of fungitoxicity as compared to little or mild toxicity developed in untreated plants and relationship between such post infection levels of toxicity in treated plants and levels of resistance induced in such stimulated high postinfection productions of fungitoxic substances in susceptible tissue may be due to a conditioning effect of treatment chemicals which make plant resistant and readily respond to infection by producing large amount of fungitoxic 
substances (Eckadt, 2011; Caballero, 2011; Bhajbhuje, 2013). As the conditioning effect wakens with time, the production of extra fungitoxic substances diminishes and the induced protective effect also declines. The salts of heavy metals, a known phytoalexin inducer chemicals, were used at dilute concentration (10-4) for wet seed treatment, most of the six heavy metal salts tested appeared to provide moderate to substantial protection to Vigna mungo against Alternaria leaf spot infection, resulting from both artificial inoculation and natural infection with virulent pathogen, Alternaria alternata Keissler. This indicated successful induction of resistance in susceptible plants by treatment with such chemicals which often persisted at appreciable levels even up to 5-6-wk. growth stage. The wet seed treatment of chemicals seemed to be the most effective, provided substantial and long persistence protection to significant level. Although apparent enhanced post-infection production of fungitoxic substance(s) was strong in Vigna mungo. Some antifungals, glycolalkaloid, phenol, ononitol, omethyl-scyllo-inositol have been reported in leaves (Battu, et al., 2011); however synthesis and accumulation of efficient phytoalexin has not so far been reported against infection of a virulent strain of leaf spot causing pathogen, Alternaria alternata Keissler, any suggestion about the possible involvement of such compounds in development of resistance is conjectural. Nevertheless, an involvement of some kind of fungitoxic substance in this process is strongly indicated. ...: .Since there is no correlation between in-vitro fungitoxicity of the phytoalexin inducer chemicals and the protection provided by them to seedlings, these chemicals may act as sensitizers, normally remain suppressed in compatible host pathogen interactions and activate the dynamic defense potential of the host and may induced series of changes in the process of metabolism of the susceptible host; appear to modify the host-parasite interactions in such a way as to result in inhibition of symptom development and an expression of host resistance. 


\section{Conclusion}

The phytoalexins are low molecular weight, anti-microbial phenol compounds that are both synthesized by, and accumulated in plants at infection site during compatible plant-pathogen interaction upon exposure to microorganism and chemicals of diverse groups. An aqueous solution at dilute conc of salts of heavy metals applied by wet seed treatment, induced significant reduction in infection and provided considerable to moderate protection to Vigna mungo (L).Hepper seedlings, at different stages against artificial inoculation with a leaf spot causing virulent pathogen, Alternaria alternata Keissler The experimental findings revealed that seed soaking in aqueous dilute solutions of chlorides of mercury, and barium to test condition provided a more vigorous defiance response to virulent pathogen as well as induced long persistent substantial protection at significant level. These phytoalexin inducer chemicals serve as very promising compounds for use in plant disease control. Wet seed treatment proved to be the most effective in seed producing plants.

\section{Acknowledgement}

The author indebted the facilitation of this work by Prof .R.P. Thakre, Mycologist and Prof. \& Head, P.G. Dept. of Botany, RTM, Nagpur University, Nagpur.

\section{Reference}

Amil-Ruiz, F., Blanco-Portales, R., Munoz-Blanco, J. and Caballero, J. L. (2011):The strawberry plant defense mechanism: A molecular review. Plant Cell Physiol., 52 (11) : 1873-1903.

Ashraf, A. and Ali, T.A. (2007): Effect of heavy metals on soil microbial community and mung beans seed germination. Pak. J. Bot., 39(2): 629636. 
Atkinson, N.J. and Urwin, P.E (2012): The interaction of plant biotic and abiotic stresses: from gene to field. Journal of Experimental Botany, 63(3): 1-21

Battu, G., Ch.K.V.L., Male, S.N.A., Priya, T.H., Malleswar and Reeshma, S.K. (2011): Phytopharmacological review of Vigna species. Pharmanest, 2(1) : 61-67.

Bhajbhuje, M.N. (2013): Role of heavy metal salts on susceptibility of Solanum melongena L. seedlings to Alternaria early blight disease. Int. J. of Life Sciences 1 (1):51-62.

Caballero J.L. (2011): The strawberry plant defence mechanism: A molecular review. Plant \& Cell Physiology,52 (11): 1873-1903.

CMI. (2010): Commonwealth Mycological Institute. Description of Pathogenic fungi and bacteria. Kew Surey, England. Pp 451-460.

Eckadt, N.A. (2011): Induction of Phytoalexin Biosynthesis: WRKY33 - Is a Target of MAPK Singling. Plant Cell, 23(4) : 1190.

Feofilova, E., Ivashechkin, A., Alekhin, A. and Sergemma, Y. (2012): Fungal spores: Dormancy, germination, chemical composition and role in biotechnology (review) Appl Biochem, \& Microbiol., 48(1): 1-34.

Iriti, M. and Franco, F. (2009): Chemical Diversity and Defence Metabolism: How Plant Cope with pathogen and ozone pollution. Int. J. Mol. Sci., 10 : 337-339.

Karthikeyan., G, Doraisamy and Rabindran, R (2009): Induction of systemic resistance in blackgram (Vigna mungo) against urdbean leaf crinckle virus by chemicals. Archives of Phytopathology and Plant protection, 42(1): $1-8$.

Mert-Turk, F. (2002): Phytoalexin: Defence or just a response to stress? J. Cell \& Mol. Biol., 1 : 1-6. 
Raju, S., Jayalaxmi, S.K., Sreeramulu, K. (2008): Comparative study on the induction of defence related enzymes in two cultivars of chickpea (Cicer arietinum L.) genotype by salicylic acid spermine and Fusarium oxysporum f. sp. Cicero. Australian Jour. Crop Sci., 2(3): 121-140.

Savithasuri (2013): Ayurveda, medicinal properties and health benefits of black gram- Vigna mungo. http://www/ayurhelp.com/plants/blackgram (Retrieved on March 20, 201)

Sharma OP, Bombawale OM, Gopali JB, Bhagat S, Yelshetty S, Singh SK, Anwar R, Singh OM. (2011). Field guide Mung bean an Urd bean. Government of India, Department of Agriculture and co-operation,NCIM ICAR, New Delhi, India.

Sobolev, V., Guo, B. and Robert, H. (2007): Interrelationship of Phytoalexin Production and Disease Resistance in Selected Peanut Genotypes. J. Agric. and Food Chem., $55: 2195-2200$

Trivedi A, Sharma Sandeep K, Hussain T, Sharma SK, Gupta PK. (2013):

Application of biodynamic preparation, bio-control agent and botanicals for organic management of virus and leaf spots of black gram (Vigna mungo L. Hepper). Acad. J. Agric. Res., 1(4): 60-64.

Wikipedia (2014):: Black gram - Vigna mungo (L) Hepper. Wikimedia Foundation, Org. en.wikipedia,org/wiki. Inc . (Retrieved on March 20, 2014).

Zhuang X, K.E. McPhee, T.E. Coram, T.L. Peever and Chilvers, M.I. (2012): Rapid transcriptome characterization and parsing of sequences in nonmodel host-pathogen interaction; pea - Sclerotinia sclerotiorum. BMC Genomics 13 : 668. Pp 1-19. 